\title{
Transfer of Downy Mildew Resistance from Wild Basil (Ocimum americanum) to Sweet Basil (O. basilicum)
}

\author{
Yariv Ben-Naim, Lidan Falach, and Yigal Cohen ${ }^{\dagger}$
}

Faculty of Life Sciences, Bar Ilan University, Ramat Gan 5290002, Israel.

Accepted for publication 28 August 2017.

\begin{abstract}
Sweet basil (Ocimum basilicum) is susceptible to downy mildew caused by the oomycete foliar pathogen Peronospora belbahrii. No resistant varieties of sweet basil are commercially available. Here, we report on the transfer of resistance gene $P b 1$ from the highly resistant tetraploid wild basil O. americanum var. americanum (PI 500945, $2 n=4 \mathrm{x}=48$ ) to the tetraploid susceptible $O$. basilicum 'Sweet basil' $(2 n=4 \mathrm{x}=48)$. F1 progeny plants derived from the interspecific hybridization PI $500945 \times$ Sweet basil were resistant, indicating that the gene controlling resistance $(\mathrm{Pbl})$ is dominant, but sterile due to the genetic distance between the parents. Despite their sterility, F1 plants were pollinated with the susceptible parent and 115 first backcross generation to the susceptible parent (BCs1) embryos were rescued in vitro. The emerging BCs1 plants segregated, upon inoculation, 5:1

of dominant genes ( $P b 1 A$ and $\left.P b 1 A^{\prime}\right)$. Thirty-one partially fertile BCs1 plants were self-pollinated to obtain BCs1-F2 or were backcrossed to Sweet basil to obtain the second backcross generation to the susceptible parent (BCs2). In total, $1 \mathrm{BCs} 1-\mathrm{F} 2$ and $22 \mathrm{BCs} 2$ progenies were obtained. The BCs1-F2 progeny segregated 35:1 resistant/susceptible, as expected from a tetraploid parent with two dominant resistant genes. The $22 \mathrm{BCs} 2$ progenies segregated 1:1 resistant/susceptible (for a BCs1 parent that carried one dominant gene for resistance) or 5:1 (for a BCs1 parent that carried two dominant genes for resistance) at a ratio of $4: 1$. The data suggest that a pair of dominant genes ( $P b 1 A$ and $\left.P b 1 A^{\prime}\right)$ residing on a two homeologous chromosomes is responsible for resistance of PI 500945 against $P$. belbahrii.
\end{abstract} resistant/susceptible, suggesting that resistance in F1 was controlled by a pair

Downy mildew, incited by the oomycete Peronospora belbahrii (Thines), is currently the most devastating foliar disease of sweet basil (Wyenandt et al. 2015). Disease control measures include a few registered fungicides (Babadoost 2010; Cohen and Rubin 2015; Farahani-Kofoet et al. 2012; Gilardi et al. 2013; Homa et al. 2014; McGrath 2016; Mersha et al. 2012, 2013; Patel et al. 2014a,b; Raid et al. 2010; Wyenandt et al. 2015). Mefenoxam, a most effective fungicide, became ineffective due to resistance of the pathogen (Cohen et al. 2013b; Pintore et al. 2016). Effective disease control may be achieved by physical measures such as nocturnal lighting (Cohen et al. 2013a), daytime solar heating (Cohen and Rubin 2015), and nocturnal fanning (Cohen and Ben-Naim 2016).

The genus Ocimum (basil) includes more than 50 species of herbs and shrubs (Paton et al. 1999; Simon et al. 1990). Interspecific hybridization and polyploidy occur commonly within this genus (Harley and Heywood 1992). The genetic relationships among the multitude number of Ocimum spp. is poorly understood (Paton and Putievsky 1996). Taxonomic issues, including morphology and chromosome number, were recently reviewed based on molecular markers and nuclear DNA content (Aghaei et al. 2012; CarovićStanko et al. 2010; Koroch et al. 2010; Rewers and Jedrzejczyk 2016; Sairkar et al. 2012).

Cytological studies revealed two basic chromosome numbers (karyotypes) in Ocimum spp.: $\mathrm{x}=8$ (Ocimum sanctum/O. gratissimum clade) and $\mathrm{x}=12$ (O. basilicum/O. americanum clade). In holy basil, O. tenuiflorum (syn. O. samctum), $2 n=4 \mathrm{x}=36$ (Carović-Stanko et al. 2010). In O. basilicum, $2 n=4 \mathrm{x}=48$ (Morton 1962; Pushpangadan and Sobti 1982; Pushpangadan et al. 1975; Vaarama 1947). In O. basilicum var. citriodorum (syn. O. americanum var. citriodorum),

†Corresponding author: Y. Cohen; E-mail: yigal.cohen1@gmail.com

This article is in the public domain and not copyrightable. It may be freely reprinted with customary crediting of the source. The American Phytopathological Society, 2018.
Additional keywords: embryo rescue, oomycete, polyploidy.
$2 n=6 \mathrm{x}=72$ (Aghaei et al. 2012; Carović-Stanko et al. 2010; Mukherjee et al. 2005; Rewers and Jedrzejczyk 2016). Interspecific hybrids are sterile due to different ploidy, homeology (lack of homology between chromosomes), or both.

Based on karyotyping and DNA content measurements, it was concluded that some $O$. americanum var. americanum accessions are diploid; some $O$. americanum var. americanum accessions, $O$. americanum var. pilosum, and $O$. basilicum are tetraploid; and lemon-type basil $O$. basilicum var. citrodorun (syn. O. americanum var. citrodorum) is hexaploid (Carović-Stanko et al. 2010; Koroch et al. 2010; Rewers and Jedrzejczyk 2016).

Plant embryos are multicellular structures that have the potential to develop into a new plant. Embryo rescue techniques enable breeders to develop interspecific hybrids (Eeckhaut et al. 2006; Miyajima 2006; Rodrangboon et al. 2002; Sage et al. 1999). The embryo can develop directly into a plantlet or indirectly, via callus tissue (Che et al. 2006; Ikeda-Iwai et al. 2003). Abortion of interspecific hybrid embryos may derive from incompatibility caused by genetic distance between parents or different ploidy (Cisneros and Tel-Zur 2010; Mehetre and Aher 2004; Trigiano and Gray 2011). No reports are available on embryo rescue in Ocimum spp.

One of the most effective measures for disease control is via genetic manipulation of the host; namely, introgression of resistant genes from another source. Potential sources of resistance against basil downy mildew (BDM) occur in wild basil species but not among commercial sweet basil entries (Farahani-Kofoet et al. 2014; Wyenandt et al. 2010). In recent studies (Ben-Naim et al. 2015a,b) we reported on 30 Ocimum spp. with various levels of resistance against BDM. Wild Ocimum spp. such as $O$. americanum var. pilosum, $O$. americanum var. americanum (syn. O. canum), O. kilimanadascharicum, $O$. gratissimum, $O$. campechianum, and $O$. tenuiflorum were highly resistant; the close relatives of $O$. basilicum (O. basilicum var. anisatum, $O$. basilicum var. thyrsiflorum, $O$. basilicum var. citrodorum, $\mathrm{O} . \times$ citrodorum, and $O$. basilicum var. minimum) were moderately resistant, while all commercial cultivars of sweet basil (O. basilicum) were highly susceptible. 
Four United States Department of Agriculture Plant Introduction (PI) entries-PI 500945, PI 500950 (belonging to O. americanum var. americanum), PI 500951, and PI 652053 (belonging to O. americanum var. pilosum) — produced highly resistant interspecific F1 hybrids when crossed with susceptible sweet basil. There is not enough data to specify whether or not known resistance in basil is race specific.

The objective of this study was to develop sweet basil resistant to BDM. This was achieved by interspecific crosses made between the resistant wild basil $O$. americanum var. americanum PI 500945 and the susceptible $O$. basilicum 'Sweet basil'. A preliminary report was recently published (Ben-Naim et al. 2016).

\section{MATERIALS AND METHODS}

Germplasm. The susceptible O. basilicum 'Sweet basil' (Genesis Seeds, Israel) and the resistant wild basil $O$. americanum var. americanum PI 500945 (Ben-Naim et al. 2015b) were used. Plants were grown in multicell trays (cell size $2.5 \mathrm{~cm}$ ) filled with a potting mixture (peat/vermiculite, 1:1 [vol/vol]), one plant per cell. Before they were sown, seed were gently scraped with a sandpaper (P 320) to improve germination. At the four- to six-leaf stage, plants were transplanted to polystyrene containers ( 1.2 by 0.5 by $0.2 \mathrm{~m})$ filled with soil mixture (as above) in a nethouse covered with 50-mesh white plastic net. During the winter season, the nethouse was covered with a transparent, infrared-impermeable, antidrip polyethylene sheet (Arava type, $100-\mu \mathrm{m}$ width; Polytiv Ltd.). Nethouses were located at Bar Ilan University (BIU) Farm ( $\left.32^{\circ} 4^{\prime} 9^{\prime \prime} \mathrm{N}, 34^{\circ} 50^{\prime} 35^{\prime \prime} \mathrm{E}\right)$.

F1 cross. Flowers of the resistant PI 500945 were emasculated and then pollinated with pollen grains taken from the susceptible Sweet basil. At 5 to 6 weeks after pollination, the F1 seed were harvested from PI 500945, dried, kept on the bench for a few weeks, and then grown as F1 progeny plants.

Embryo rescue. F1 plants grown in a nethouse were all resistant to BDM but sterile, producing no seed. Therefore, the embryo rescue technique developed for Lycopersicon (Doganlar et al. 1997) was employed to obtain progeny plants from these F1 plants. The sterile F1 plants were pollinated with Sweet basil pollen. The pollinated flowers, possibly containing fertilized ovules, were collected 5 to 8 days post pollination, disinfected, and cultured in 5.5-cm Petri dishes containing Murashige and Skoog (MS) culture medium. The disinfection process was carried out as follows. Flowers were flushed with distilled water for $2 \mathrm{~h}$ and placed on sterile filter paper, and petals were removed carefully. The receptacle, carrying four immature seeds (nutlets), was separated from the pedicel, placed in $0.3 \%$ hypochlorite solution for $15 \mathrm{~min}$, washed with sterile water, placed for $5 \mathrm{~min}$ in ethanol $70 \%$, and rinsed three times with double-distilled sterile water. The cut end of the receptacle was placed on MS medium (Murashige and Skoog 1962) containing $100 \mathrm{mg}$ of myo-inositol,
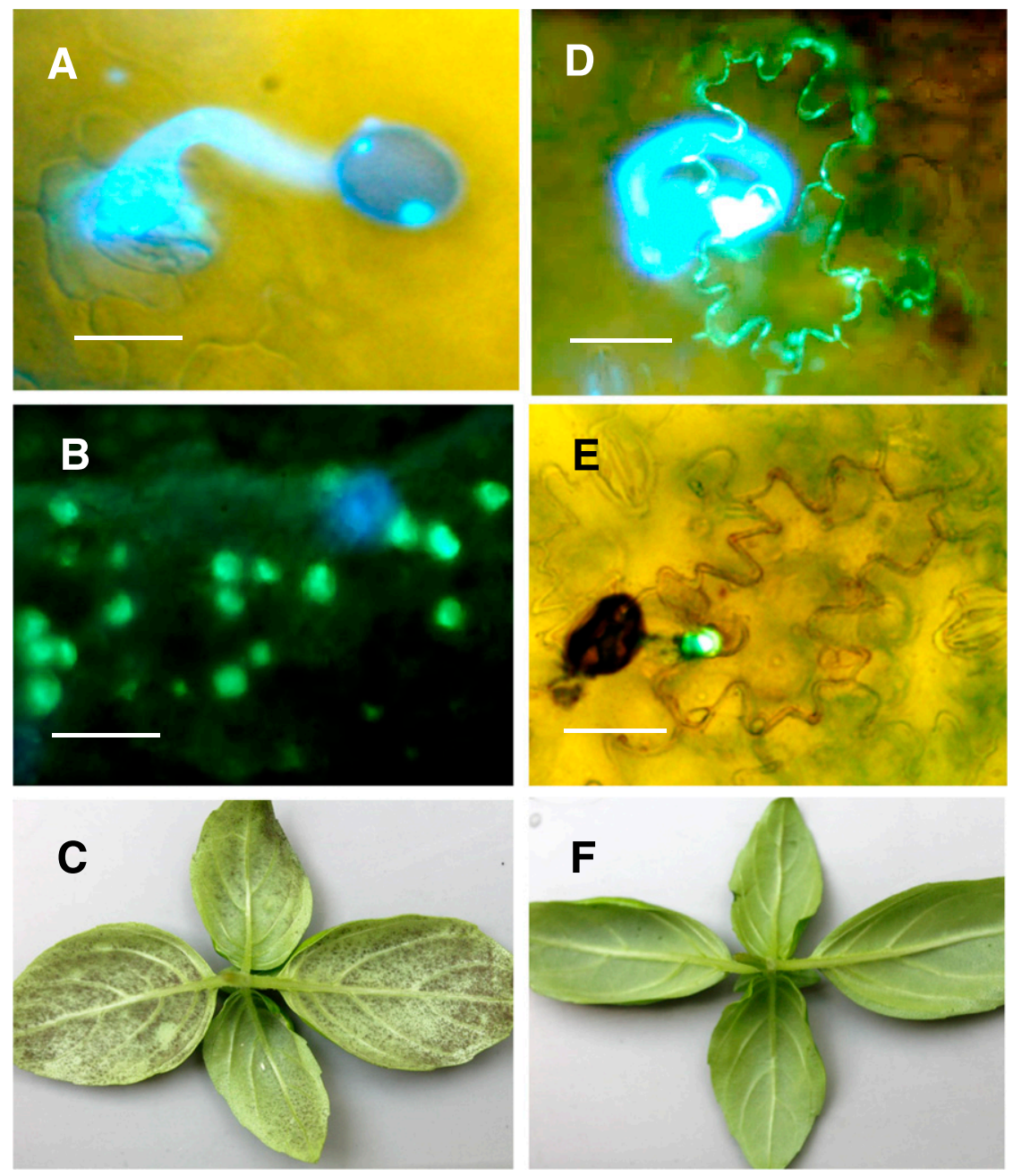

Fig. 1. A, B, D, and E, Fluorescent and $\mathbf{C}$ and $\mathbf{F}$, regular micrographs showing the development of Peronospora belbahrii in susceptible (A to C) and resistant (D to F) Ocimum spp. A to D: Bar $=30 \mu \mathrm{m}$. A, Spore germination and germ-tube penetration into a leaf of the susceptible Ocimum basilicum 'Sweet basil' at 1 day postinoculation (dpi). B, Haustoria, fluorescing green, in the mesophyll of Sweet basil at 7 dpi. C, Sporulation on the lower leaf surface of Sweet basil at 7 dpi. $\mathbf{D}$ and $\mathbf{E}$, Spore germination and germ-tube penetration into a leaf of the resistant $O$. americanum var. americanum PI 500945 at 1 dpi. Note the massive callose encasement of the epidermal cell in D and the hypersensitive response in E. F, No sporulation occurs at 7 dpi on leaves PI 500945. Leaves in A, B, D, and E were stained with basic aniline blue pH8.9 and calcofluor. 
$0.4 \mathrm{mg}$ of thiamine- $\mathrm{HCl}, 30 \mathrm{~g}$ of sucrose, and $8 \mathrm{~g}$ of plant agar per liter ( $\mathrm{pH}$ 5.8) (Duchefa Biochemicals). The Petri dishes were incubated at $25^{\circ} \mathrm{C}$ in the dark for 2 weeks and then at $18^{\circ} \mathrm{C}(12 \mathrm{~h} /$ day, $45 \mu \mathrm{mol} \mathrm{m} \mathrm{m}^{-2} \mathrm{~s}^{-1}$ ) for another 2 weeks. After 4 weeks of incubation, some ovules developed seed. They were transferred onto MS medium amended with $6 \mathrm{mg}$ of I-inositol, $20 \mathrm{~g}$ of sucrose, and $2 \mathrm{mg}$ of 6-benzylaminopurine per liter and incubated at $18^{\circ} \mathrm{C}(12 \mathrm{~h} /$ day, $45 \mu \mathrm{mol} \mathrm{m} \mathrm{m}^{-2} \mathrm{~s}^{-1}$ ). After 20 to 30 days, small plantlets, most of them with no roots, had developed. Plantlets were transferred to 5.5-cm Petri dishes (1 to 2 plantlets/dish) containing rooting medium made of MS salts amended with $1 \mathrm{mg}$ of thiamine $\cdot \mathrm{HCl}, 100 \mathrm{mg}$ of I-inositol, $30 \mathrm{~g}$ of sucrose, and $1 \mathrm{mg}$ of naphthaleneacetic acid per liter. Plates were incubated at $27^{\circ} \mathrm{C}\left(16 \mathrm{~h} /\right.$ day, $\left.45 \mu \mathrm{mol} \mathrm{m}^{-2} \mathrm{~s}^{-1}\right)$ until plantlets developed lateral roots ( 1 to 3 weeks). The plants were transplanted into Jiffy pots (Jiffy-7, Peat Pellets and Coco Pellet; https://www.jiffygroup.com/) for acclimation (1 week) and then planted in the nethouse.

Pathogen. Twenty-four isolates of Peronospora belbahrii were used in this study. They were collected during 2012 to 2017 (March to November) from the four major growing regions in Israel (Shean Valley in the north, Southern Jordan River Valley, Central Coastal Plain, and the Bsor region in southwest). An isolate was defined by the location and date of collection. The isolates collected during 2012 were sensitive to mefenoxam whereas those collected during 2013 to 2017 were all resistant to this fungicide (Cohen et al. 2013a) (unpublished data). Sweet basil was highly susceptible to all isolates whereas PI 500945 was resistant, showing no symptoms with any isolate used (Ben-Naim et al. 2015b). The mefenoxam-resistant isolate Knafo-3 (collected in 2013 at Ein-Tamar, Southern Jordan Valley) was used in all experiments described below. It was maintained by repeated inoculations of potted Sweet basil plants in a growth chamber at $25^{\circ} \mathrm{C}$.

Inoculation and disease assessment in growth chambers. Fresh spores of $P$. belbahrii were collected from infected plants into cold distilled water, their number adjusted to 5,000 spores $/ \mathrm{ml}$, and spray inoculated onto the upper leaf surfaces of the test plants (six- to eight-leaf stage) with the aid of a fine glass atomizer. Inoculated plants were incubated in a dew chamber at $18^{\circ} \mathrm{C}$ in the dark for $15 \mathrm{~h}$ to ensure infection and thereafter for 6 days at $25^{\circ} \mathrm{C}$ under continuous illumination $\left(60 \mu \mathrm{mol} \mathrm{m} \mathrm{m}^{2} \mathrm{~s}^{-1}\right)$ to allow symptom production. Plants were returned to the dew chamber on the seventh day postinoculation (dpi) to enable sporulation of the pathogen on leaves of the inoculated plants. Each plant was visually inspected for disease symptoms and sporulation of the pathogen. Plants showing symptoms or sporulation were considered susceptible $(\mathrm{S})$ whereas plants showing neither symptoms nor sporulation were considered resistant $(\mathrm{R})$.

Inoculation and disease assessment in the field. The inoculated parental and progeny plants ( $\mathrm{S}$ and $\mathrm{R}$ ) were transplanted to a nethouse ( 6 by $50 \mathrm{~m}$ ) at the BIU Farm. At 7 days after planting, when they had reached the 10- to 12-leaf stage, plants were spray inoculated with spore suspension $(5,000 \mathrm{spores} / \mathrm{ml})$ of $P$. belbahrii with the aid of a hand sprayer. Inoculation took place at 8:00 P.M. to ensure high humidity for infection. Starting at 1 week after inoculation, disease records ( $\mathrm{S}$ and $\mathrm{R}$, as described above) were taken from the inoculated plants.
DNA content in Ocimum nuclei by flow cytometry. Plants were grown in the greenhouse. Leaves number 6 to 8 from the stem base were excised from 10-leaf plants, placed in aluminum foil, and frozen in liquid nitrogen. The tissue was gently crushed using a mortar and pestle, transferred into a 50-ml tube, and kept on ice for approximately 10 min until thawing of the tissue. Buffer A (see below) was added to cover the tissue and the tube was shaken for $30 \mathrm{~min}$. The extract was transferred to a new tube through a $40-\mu \mathrm{m}$ mesh sieve strainer (BD Falcon) and centrifuged for $1 \mathrm{~min}$ at $11,000 \times g$. The supernatant was discarded and the pellet was suspended in $1.5 \mathrm{ml}$ of buffer B (see below). The filtration through strainer was repeated and the filtrate was kept at room temperature for 30 min for DNA staining with propidium iodide (see below). DNA content in the nuclei was measured $(n=3)$ by using a FACScan Flow Cytometer (Becton Dickinson Immunocytometry Systems caliber) equipped with argon-ion laser emitting at $488 \mathrm{~nm}$. Only measurements having covariance value of $\leq 5 \%$ were used (Doležel et al. 2007). As a reference, we used nuclei of watermelon (Citrullus lanatus var. lanatus), which have a $2 \mathrm{C}$ value of 0.88 to $0.98 \mathrm{pg}=900 \mathrm{Mbp}$ (Arumuganathan and Earle 1991; Zhang et al. 1994). DNA content was converted into picograms per nucleus by using the formula $1 \mathrm{pg}=978 \mathrm{Mbp}$ (Dolezel et al. 2003). After an initial calibration, Sweet basil nuclei served as a reference.

Preparation of nuclei was done according to Arumuganathan and Earle (1991), with modification. The following solutions were used: $\mathrm{MgSO}_{4}$ buffer $=10 \mathrm{mM} \mathrm{MgSO}{ }_{4} \cdot 7 \mathrm{H}_{2} \mathrm{O}, 50 \mathrm{mM} \mathrm{KCl}$, and $5 \mathrm{mM}$ HEPES (adjusted to $\mathrm{pH} 8.0$ ); extraction buffer $\mathrm{A}=\mathrm{MgSO}_{4}$ buffer amended with $1 \%$ (wt/vol) polyvinylpyrrolidone, $6.5 \mathrm{mM}$ dithiothreitol (DTT), and $0.25 \%$ (vol/vol) Triton X-100 (stored at $4{ }^{\circ} \mathrm{C}$ ); and extraction buffer $\mathrm{B}=\mathrm{MgSO}_{4}$ buffer amended with $6.5 \mathrm{mM}$ DTT, $0.25 \%$ (vol/vol) Triton X-100, propidium iodide (Acros Organics) at $0.2 \mathrm{mg} / \mathrm{ml}$, and RNase (DNase-free) at $1.25 \mu \mathrm{g} / \mathrm{ml}$ (prepared on ice just prior to use).

Microscopy. In three different experiments, leaf discs (12 $\mathrm{mm}$ in diameter) were removed from leaves 6 to 8 of 10-leaf inoculated plants at 1 and $7 \mathrm{dpi}$. Discs were clarified in boiling ethanol for $10 \mathrm{~min}$, placed in basic aniline blue solution $(0.05 \%$ aniline blue in $0.05 \mathrm{M} \mathrm{K}_{2} \mathrm{HPO}_{4}, \mathrm{pH} 8.9$ ) at $4^{\circ} \mathrm{C}$ for $24 \mathrm{~h}$, stained with $0.01 \%$ calcofluor (Sigma), and examined with an Olympus A70 epi-fluorescent microscope.

Statistics. DNA content values were compared by analysis of variance using JMP software (SAS Institute). Significant differences between means $(n=3)$ were performed using Tukey's honestly significant difference test with $\alpha=0.05$ (honest significant difference).

The Excel program was used to perform $\chi^{2}$ tests. $P$ values $\geq 0.05$ indicated acceptance of the suggested inheritance model.

\section{RESULTS}

Microscopy of resistance. Potted PI 500945 (O. americanum var. americanum) plants, inoculated in growth chambers with any of the 24 isolates collected during 2012 to 2017, showed no disease symptoms (full resistance) until 10 days after inoculation. Similarly

TABLE 1. DNA content (mean and standard deviation [SD] of the mean) in Ocimum spp. as determined by flow cytometry

\begin{tabular}{|c|c|c|c|c|c|}
\hline Species & Accession & Content (pg/nucleus) & SD & Statistic & Ploidy \\
\hline O. americanum var. americanum & PI 253158 & 2.05 & 0.06 & e & $2 n=2 x=24$ \\
\hline O. basilicum & 'Sweet basil & 4.66 & 0.05 & $\mathrm{~d}$ & $2 n=4 x=48$ \\
\hline O. basilicum & 'Aroma 2' & 4.61 & 0.03 & $\mathrm{~d}$ & $2 n=4 x=48$ \\
\hline O. basilicum & PI 652070 & 4.63 & 0.04 & $\mathrm{~d}$ & $2 n=4 x=48$ \\
\hline O. basilicum & 'Dark Opal' & 4.58 & 0.03 & $\mathrm{~cd}$ & $2 n=4 x=48$ \\
\hline O. basilicum var. minimum & PI 170579 & 4.42 & 0.02 & $\mathrm{c}$ & $2 n=4 x=48$ \\
\hline O. americanum var. americanum & PI 500945 & 4.41 & 0.06 & $\mathrm{c}$ & $2 n=4 x=48$ \\
\hline O. basilicum var. citrodorum & 'Mrs. Burns' & 7.52 & 0.02 & $\mathrm{~b}$ & $2 n=6 x=72$ \\
\hline O. basilicum var. citrodorum & 'Lemon basil' & 7.5 & 0.02 & $\mathrm{~b}$ & $2 n=6 x=72$ \\
\hline O. basilicum var. anisatum & PI 172997 & 10.41 & 0.01 & $\mathrm{a}$ & $2 n=8 x=96$ \\
\hline O. basilicum var. anisatum & PI 172998 & 10.45 & 0.04 & a & $2 n=8 x=96$ \\
\hline
\end{tabular}


inoculated Sweet basil plants showed abundant chlorotic symptoms at $7 \mathrm{dpi}$ with massive sporulation with all isolates. Adult PI 500945 plants grown in nethouses or plastic-houses during seven seasons (2014 to 2017) were resistant to BDM whereas Sweet basil plants were highly susceptible.

The microscopic responses of the susceptible Sweet basil and the resistant PI 500945 to inoculation with isolate Knafo 3 at 1 and 7 dpi are shown in Figure 1. In Sweet basil at $1 \mathrm{dpi}$, spores of P. belbahrii germinated, produced an appressorium, and penetrated into the epidermis via the stomatal opening. No response of the penetrated epidermal cell was observed (Fig. 1A). At 7 dpi, abundant haustoria were seen inside the mesophyll (Fig. 1B) and massive sporulation (approximately $1 \times 10^{4}$ spores $/ \mathrm{mm}^{2}$ ) occurred on the lower leaf surface (Fig. 1C). In the resistant PI 500945 at 1 dpi, spores germinated and penetrated equally well into the epidermal cells but the penetrated cells showed massive accumulation of callose along their cell walls (Fig. 1D). The content of the penetrated cell became dark, producing a hypersensitive response (Fig. 1E). The pathogen stopped developing when the primary vesicle has developed in the epidermal cell (Fig. 1E, yellow-fluorescing spot). A week after inoculation, neither sporophores nor spores were detected in PI 500945 (Fig. 1F). Leaves of F1 plants (PI $500945 \times$ Sweet basil) showed microscopic responses similar to those of the resistant parent PI 500945 (not shown).

DNA content. Flow cytometry data are shown in Table 1. Based on the 2C nuclear DNA content (picograms per nucleus), Ocimum spp. were found to belong to either one of four ploidy types:

O. americanum var. americanum (syn. O. canum), diploid $(2 n=$ $2 \mathrm{x}=24)$

O. basilicum cultivars, tetraploid $(2 n=4 \mathrm{x}=48)$;
O. basilicum var. minimum, tetraploid $(2 n=4 \mathrm{x}=48)$;

O. americanum var. americanum, tetraploid $(2 n=4 \mathrm{x}=48)$;

O. basilicum var. citrodorum, hexaploid $(2 n=6 \mathrm{x}=72)$;

O. basilicum var. anisatum, octaploid $(2 n=8 \mathrm{x}=96)$.

DNA contents in each ploidy type $(2 \mathrm{x}, 4 \mathrm{x}, 6 \mathrm{x}$, and $8 \mathrm{x})$ were significantly different from each other. The six $4 \mathrm{x}$ entries showed three levels of statistical difference (Table 1).

These results corroborate the findings of previous studies (Koroch et al. 2010; Rewers and Jedrzejczyk 2016). The data suggest that the parental lines used in this study ( $O$. basilicum 'Sweet basil' and $O$. americanum var. americanum PI 500945) carry the same number of chromosomes $(2 n=4 \mathrm{x}=48)$. The fact that they produce a sterile F1 hybrid (see below) suggests that they carry homeologous chromosomes.

Inheritance of resistance. F1 plants of the cross PI $500945 \times$ Sweet basil were fully resistant to all 24 isolates used in this study (data not shown). Because such F1 plants were sterile (produced no pollen grains, indicating male sterility, and failed to cross with viable pollen of Sweet basil, indicating female sterility), no selfpollination could take place to produce the F2 generation. To explore the mode of inheritance of resistance, F1 plants were pollinated with pollen grains taken from susceptible Sweet basil. The first backcross generation to the susceptible parent (BCs1) progeny plants were obtained by using embryo rescue techniques. In total, 115 BCs1 plants were rescued from approximately 7,000 flowers (approximately 28,000 ovules). The response to downy mildew of the susceptible parent Sweet basil, the resistant parent PI 500945, their F1 plants, and their BCs1 progeny is shown in Table 2. All 46 $\mathrm{F} 1$ plants were fully resistant to the disease. $\mathrm{BCs} 1$ plants segregated $100: 15 \mathrm{R} / \mathrm{S}$. A $\chi^{2}$ analysis of four models (one dominant, two

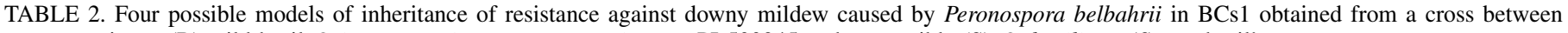
parents resistant (R) wild basil Ocimum americanum var. americanum PI 500945 and susceptible (S) O. basilicum 'Sweet basil'

\begin{tabular}{|c|c|c|c|c|c|c|c|c|c|}
\hline \multirow[b]{2}{*}{ Pedigree } & \multirow[b]{2}{*}{$N^{\mathrm{a}}$} & \multicolumn{2}{|c|}{ Observed ratio } & \multicolumn{2}{|c|}{ Expected ratio } & \multirow[b]{2}{*}{ Tested ratio } & \multirow[b]{2}{*}{ Genes $^{b}$} & \multirow[b]{2}{*}{$P^{\mathrm{c}}$} & \multirow[b]{2}{*}{$\chi^{2}$} \\
\hline & & $\mathrm{R}$ & $\mathrm{S}$ & $\mathrm{R}$ & $\mathrm{S}$ & & & & \\
\hline PI 500945 (R) & 20 & 20 & $\ldots$ & $\ldots$ & $\ldots$ & $\ldots$ & $\ldots$ & $\ldots$ & $\ldots$ \\
\hline Sweet basil (S) & 20 & $\ldots$ & 20 & $\ldots$ & $\ldots$ & $\ldots$ & $\ldots$ & $\ldots$ & $\ldots$ \\
\hline $\mathrm{R} \times \mathrm{S}, \mathrm{F} 1$ & 46 & 46 & $\ldots$ & $\ldots$ & $\ldots$ & $\ldots$ & $\ldots$ & $\ldots$ & $\ldots$ \\
\hline BCs1 & 115 & 100 & 15 & 57.5 & 57.5 & $1: 1$ & $1 \mathrm{D}$ & $2.258 \mathrm{E}-15$ & $8.008 \mathrm{E}-30$ \\
\hline BCs1 & 115 & 100 & 15 & 86 & 29 & $3: 1$ & $2 \mathrm{D}$ & 0.0026 & $1.099 \mathrm{E}-05$ \\
\hline BCs1 & 115 & 100 & 15 & 96 & 19 & $5: 1$ & $1 \mathrm{DD}$ & $0.3162 *$ & 0.165 \\
\hline BCs1 & 115 & 100 & 15 & 109 & 6 & $19: 1$ & $1 \mathrm{TD}$ & 0.00016 & 4.054E-08 \\
\hline
\end{tabular}

a Number of plants.

b $\mathrm{D}=$ dominant, $\mathrm{DD}=$ duplicate dominant, and $\mathrm{TD}=$ triplicate dominant

c Asterisk (*) indicates accepted $(P>0.05)$.

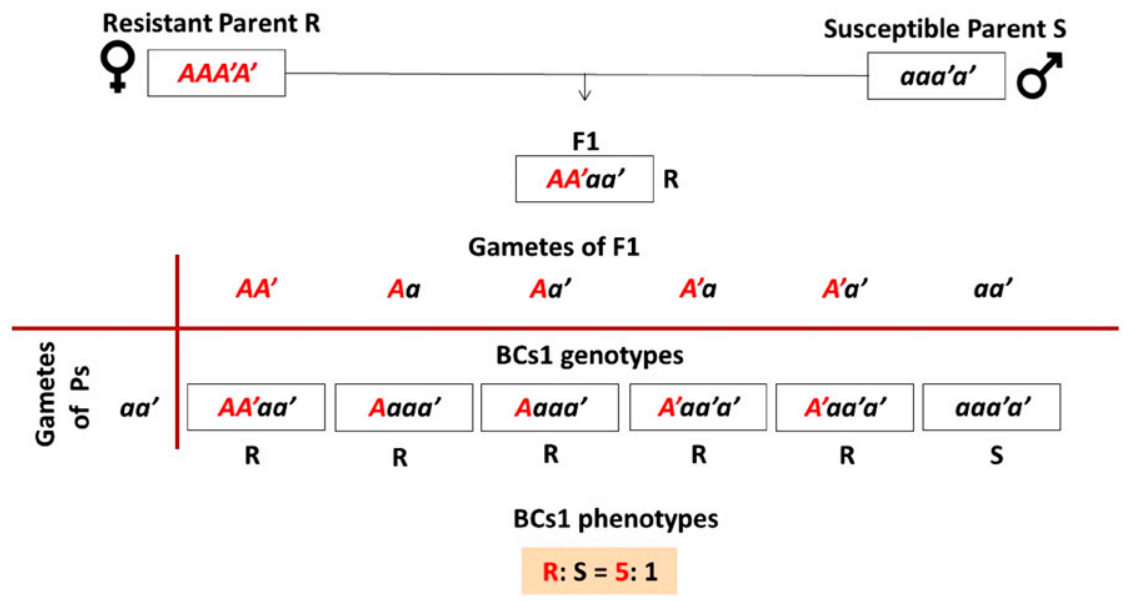

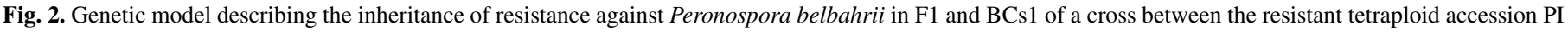
500945 of Ocimum americanum var. americanum and the susceptible tetraploid $O$. basilicum 'Sweet basil'. 


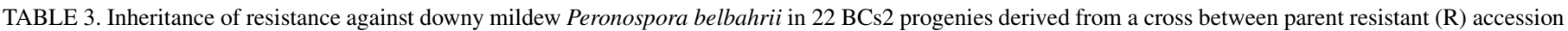
PI 500945 of Ocimum americanum var. americanum and susceptible (S) O. basilicum 'Sweet basil'

\begin{tabular}{|c|c|c|c|c|c|c|c|c|c|}
\hline \multirow[b]{2}{*}{ Pedigree } & \multirow[b]{2}{*}{$N^{\mathrm{a}}$} & \multicolumn{2}{|c|}{ Observed ratio } & \multicolumn{2}{|c|}{ Expected ratio } & \multirow[b]{2}{*}{ Tested ratio } & \multirow[b]{2}{*}{ Genes $^{b}$} & \multirow[b]{2}{*}{$P$} & \multirow[b]{2}{*}{$\chi^{2}$} \\
\hline & & $\mathrm{R}$ & $\mathrm{S}$ & $\mathrm{R}$ & $\mathrm{S}$ & & & & \\
\hline PI 500945 (R) & 17 & 17 & 0 & $\ldots$ & $\ldots$ & $\ldots$ & $\ldots$ & $\ldots$ & $\ldots$ \\
\hline 'Sweet basil (S) & 48 & 0 & 48 & $\ldots$ & $\cdots$ & $\ldots$ & $\ldots$ & $\ldots$ & $\ldots$ \\
\hline $\mathrm{R} \times \mathrm{S}, \mathrm{F} 1$ & 25 & 25 & 0 & $\ldots$ & $\ldots$ & $\ldots$ & $\ldots$ & $\ldots$ & $\ldots$ \\
\hline $\mathrm{BCs} 2(1)$ & 46 & 34 & 12 & 38 & 8 & $5: 1$ & $1 \mathrm{DD}$ & 0.120 & 0.023 \\
\hline $\mathrm{BCs} 2(2)$ & 23 & 11 & 12 & 11.5 & 11.5 & $1: 1$ & $1 \mathrm{D}$ & 0.835 & 1.926 \\
\hline $\mathrm{BCs} 2(3)$ & 21 & 9 & 12 & 10.5 & 10.5 & $1: 1$ & $1 \mathrm{D}$ & 0.513 & 0.482 \\
\hline $\mathrm{BCs} 2(4)$ & 28 & 16 & 12 & 14 & 14 & $1: 1$ & $1 \mathrm{D}$ & 0.450 & 0.357 \\
\hline $\mathrm{BCs} 2(6)$ & 31 & 16 & 15 & 15.5 & 15.5 & $1: 1$ & $1 \mathrm{D}$ & 0.857 & 2.150 \\
\hline $\mathrm{BCs} 2(7)$ & 24 & 13 & 11 & 12 & 12 & $1: 1$ & $1 \mathrm{D}$ & 0.683 & 1.002 \\
\hline BCs2(9) & 8 & 9 & 1 & 7.5 & 2.5 & $5: 1$ & $1 \mathrm{DD}$ & 0.273 & 0.122 \\
\hline BCs2(10) & 29 & 11 & 17 & 14.5 & 14.5 & $1: 1$ & $1 \mathrm{D}$ & 0.259 & 0.109 \\
\hline $\mathrm{BCs} 2(12)$ & 27 & 15 & 12 & 13.5 & 13.5 & $1: 1$ & $1 \mathrm{D}$ & 0.564 & 0.606 \\
\hline BCs2(13) & 17 & 13 & 4 & 14 & 3 & $5: 1$ & $1 \mathrm{DD}$ & 0.525 & 0.509 \\
\hline BCs2(15) & 43 & 13 & 30 & 21.5 & 21.5 & $1: 1$ & $1 \mathrm{D}$ & $0.010^{c}$ & 0.00014 \\
\hline $\mathrm{BCs} 2(16)$ & 36 & 21 & 15 & 18 & 18 & $1: 1$ & $1 \mathrm{D}$ & 0.317 & 0.167 \\
\hline BCs2(17) & 19 & 12 & 7 & 9.5 & 9.5 & $1: 1$ & $1 \mathrm{D}$ & 0.251 & 0.103 \\
\hline BCs2(19) & 21 & 16 & 5 & 17 & 4 & $5: 1$ & $1 \mathrm{DD}$ & 0.578 & 0.646 \\
\hline $\mathrm{BCs} 2(20)$ & 34 & 21 & 13 & 17 & 17 & $1: 1$ & $1 \mathrm{D}$ & 0.170 & 0.046 \\
\hline BCs $2(21)$ & 17 & 12 & 5 & 14 & 3 & $5: 1$ & $1 \mathrm{DD}$ & 0.203 & 0.066 \\
\hline BCs2(22) & 22 & 13 & 9 & 11 & 11 & $1: 1$ & $1 \mathrm{D}$ & 0.394 & 0.266 \\
\hline $\mathrm{BCs} 2(24)$ & 15 & 9 & 6 & 7.5 & 7.5 & $1: 1$ & $1 \mathrm{D}$ & 0.439 & 0.337 \\
\hline BCs2(26) & 43 & 23 & 20 & 21.5 & 21.5 & $1: 1$ & $1 \mathrm{D}$ & 0.647 & 0.864 \\
\hline BCs2(29) & 28 & 12 & 16 & 14 & 14 & $1: 1$ & $1 \mathrm{D}$ & 0.450 & 0.357 \\
\hline BCs $2(30)$ & 26 & 15 & 11 & 13 & 13 & $1: 1$ & $1 \mathrm{D}$ & 0.433 & 0.327 \\
\hline $\mathrm{BCs} 2(31)$ & 37 & 20 & 17 & 18.5 & 18.5 & $1: 1$ & $1 \mathrm{D}$ & 0.622 & 0.777 \\
\hline$(B C s 1-1) \times(B C s 1-1)^{d}$ & 29 & 27 & 2 & 28 & 1 & $35: 1$ & $1 \mathrm{DD}$ & 0.309 & 0.158 \\
\hline
\end{tabular}

a Number of plants.

b $\mathrm{D}=$ dominant and DD = duplicate dominant.

c A single pedigree for which none of the models was accepted $(P<0.05)$.

d Self-pollinated pedigree of a fertile BCs1-1.

BCs1 genotypes

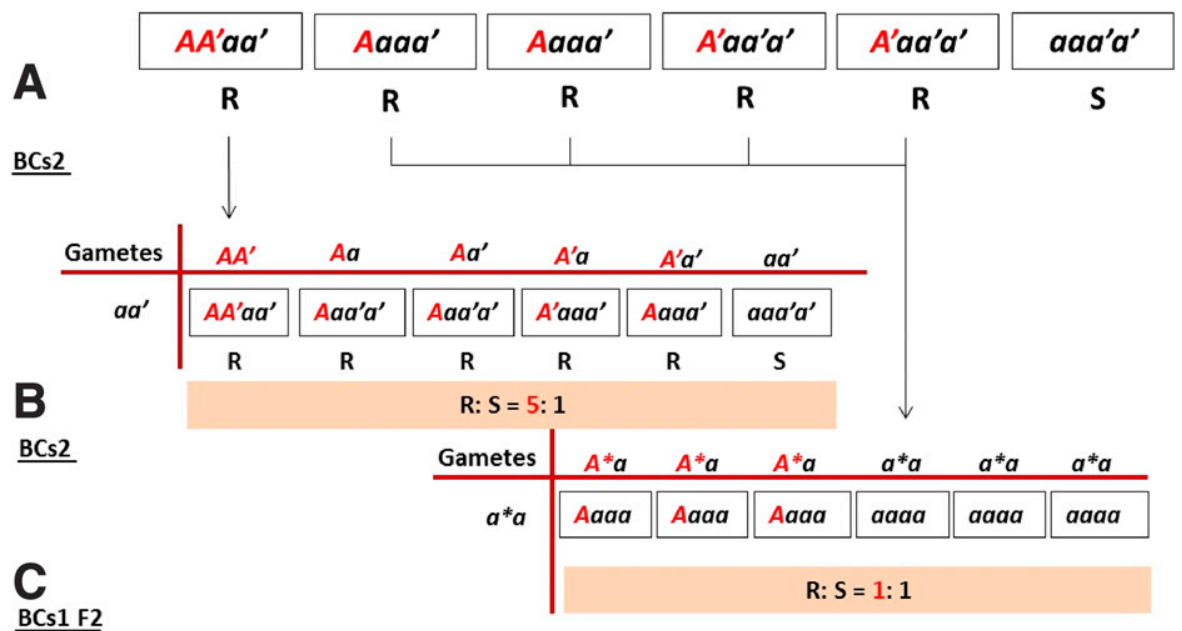

\begin{tabular}{|c|c|c|c|c|c|c|}
\hline Gametes & $A A^{\prime}$ & $A a$ & $A a^{\prime}$ & $A^{\prime} a$ & $A^{\prime} a^{\prime}$ & $a a^{\prime}$ \\
\hline$A A^{\prime}$ & $A A^{\prime} A A^{\prime}$ & $A A^{\prime} A a$ & $A A^{\prime} A a^{\prime}$ & $A A^{\prime} A^{\prime} a$ & $A A^{\prime} A^{\prime} a^{\prime}$ & $A A^{\prime} a a^{\prime}$ \\
\hline$A a$ & $A a A^{\prime} a^{\prime}$ & $A a A a$ & $A a A a^{\prime}$ & $A a A^{\prime} a$ & $A a^{\prime} A^{\prime} a^{\prime}$ & Aaaa' $^{\prime}$ \\
\hline$A a^{\prime}$ & $A a^{\prime} A A^{\prime}$ & $A a^{\prime} A a$ & $A a^{\prime} A a^{\prime}$ & $A a^{\prime} A^{\prime} a$ & $A a^{\prime} A^{\prime} a^{\prime}$ & $A a^{\prime} a a^{\prime}$ \\
\hline$A^{\prime} a$ & $A^{\prime} a A A^{\prime}$ & $A^{\prime} a A a$ & $A^{\prime} a A a^{\prime}$ & $A^{\prime} a A^{\prime} a$ & $A^{\prime} a A^{\prime} a^{\prime}$ & $A^{\prime} a a a^{\prime}$ \\
\hline$A^{\prime} a^{\prime}$ & $A^{\prime} a^{\prime} A A^{\prime}$ & $A^{\prime} a^{\prime} A a$ & $A^{\prime} a^{\prime} A a^{\prime}$ & $A^{\prime} a^{\prime} A^{\prime} a$ & $A^{\prime} a^{\prime} A^{\prime} a^{\prime}$ & $A^{\prime} a^{\prime} a a^{\prime}$ \\
\hline$a a^{\prime}$ & $a a^{\prime} A A^{\prime}$ & $a a^{\prime} A a$ & $a a^{\prime} A a^{\prime}$ & $a a^{\prime} A^{\prime} a$ & $a a^{\prime} A^{\prime} a^{\prime}$ & $a a^{\prime} a a^{\prime}$ \\
\hline
\end{tabular}

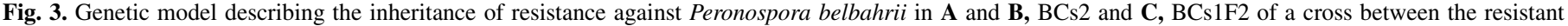
tetraploid accession PI 500945 of Ocimum americanum var. americanum and the susceptible tetraploid O. basilicum 'Sweet basil'. 


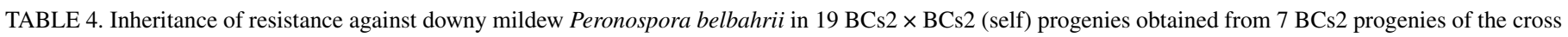
between resistant (R) accession Ocimum americanum var. americanum PI 500945 and susceptible (S) O. basilicum 'Sweet basil'

\begin{tabular}{|c|c|c|c|c|c|c|c|}
\hline \multirow[b]{2}{*}{ BCs1 } & \multirow[b]{2}{*}{$\mathrm{BCs} 2 \times \mathrm{BCs} 2$ (self) } & \multirow[b]{2}{*}{$N^{\mathrm{a}}$} & \multicolumn{2}{|c|}{ Observed ratio } & \multicolumn{2}{|c|}{ Expected ratio } & \multirow[b]{2}{*}{$P$ for $3: 1^{\mathrm{b}}$} \\
\hline & & & $\mathrm{R}$ & $\mathrm{S}$ & $\mathrm{R}$ & $\mathrm{S}$ & \\
\hline \multirow[t]{10}{*}{1} & 2 & 10 & 8 & 2 & 7.5 & 2.5 & 0.715 \\
\hline & 3 & 30 & 19 & 11 & 22.5 & 7.5 & 0.140 \\
\hline & 5 & 22 & 15 & 7 & 16.5 & 5.5 & 0.460 \\
\hline & 15 & 10 & 10 & 0 & 7.5 & 2.5 & 0.068 \\
\hline & 16 & 12 & 9 & 3 & 9 & 3 & 1 \\
\hline & 20 & 13 & 10 & 3 & 9.75 & 3.25 & 0.872 \\
\hline & 22 & 40 & 34 & 6 & 30 & 10 & 0.145 \\
\hline & 27 & 57 & 43 & 14 & 42.75 & 14.25 & 0.940 \\
\hline & 29 & 83 & 59 & 24 & 62.25 & 20.75 & 0.410 \\
\hline & 30 & 27 & 17 & 10 & 20.25 & 6.75 & 0.149 \\
\hline 2 & 8 & 17 & 11 & 6 & 12.75 & 4.25 & 0.327 \\
\hline \multirow[t]{3}{*}{3} & 1 & 49 & 47 & 2 & 37 & 12 & $0.00071 *$ \\
\hline & 5 & 14 & 12 & 2 & 10.5 & 3.5 & 0.355 \\
\hline & 10 & 2 & 2 & 0 & 1.5 & 0.5 & 0.414 \\
\hline 4 & 3 & 21 & 15 & 6 & 15.75 & 5.25 & 0.705 \\
\hline 6 & 3 & 15 & 11 & 4 & 11.25 & 3.75 & 0.882 \\
\hline 17 & 1 & 2 & 1 & 1 & 1.5 & 0.5 & 0.414 \\
\hline \multirow[t]{2}{*}{26} & 3 & 6 & 4 & 2 & 4.5 & 1.5 & 0.640 \\
\hline & 1 & 7 & 4 & 3 & 5.25 & 1.75 & 0.276 \\
\hline
\end{tabular}

a Number of plants.

b Asterisk (*) indicates unaccepted $(P<0.05)$ for 3:1 but accepted for 35:1 $(P=0.57)$.

\begin{tabular}{|c|c|c|c|c|c|c|}
\hline \multicolumn{7}{|c|}{$\mathrm{BCs} 2 \times \mathrm{BCs} 2(\mathrm{~F} 2)$} \\
\hline Gametes & $A^{*} a$ & $A^{*} \boldsymbol{a}$ & $A^{*} \boldsymbol{a}$ & $a^{*} a$ & $a^{*} a$ & $a^{*} a$ \\
\hline$A^{*} a$ & AAaa & AAaa & Aaaa & Aaaa & Aaaa & Aaaa \\
\hline$A^{*} a$ & AAaa & AAaa & AAaa & Aaaa & Aaaa & Aaaa \\
\hline$A^{*} a$ & AAara & AAaa & AAaa & Aaaa & Aaaa & Aaaa \\
\hline$a^{*} a$ & Aaaa & Aaaa & Aaaa & aaaa & aaaa & aaaa \\
\hline$a^{*} a$ & Aaaa & Aaaa & Aaaa & aaaa & aaaa & aaaa \\
\hline$a^{*} a$ & Aaaa & Aaaa & Aaaa & aaaa & aaaa & aaaa \\
\hline $\begin{array}{l}{ }_{A}^{*} A=A \text { or } A \\
\text { and } a=a \text { or } a,\end{array}$ & & & R: $S$ & $3: 1$ & & \\
\hline
\end{tabular}

\begin{tabular}{|c|c|c|c|c|c|c|}
\hline \multicolumn{7}{|c|}{ BCs2 X "Sweet basil" (BCs3) } \\
\hline Gametes & $A^{*} a$ & $A^{*} a$ & $A^{*} a$ & $a^{*} a$ & $a^{*} a$ & $a^{*} a$ \\
\hline$a^{*} a$ & Aaaa & Aaaa & Aaaa & aaaa & aaaa & aaaa \\
\hline$a^{*} a$ & Aaaa & Aaaa & Aaaa & aaaa & aaaa & aaaa \\
\hline$a^{*} a$ & Aaaa & Aaaa & Aaaa & aaaa & aaaa & aaaa \\
\hline$a^{*} a$ & Aaaa & Aaaa & Aaaa & aaaa & aaaa & aaaa \\
\hline$a^{*} a$ & Aaaa & Aaaa & Aaaa & aaaa & aaaa & aaaa \\
\hline$a^{*} a$ & Aaaa & Aaaa & Aaaa & aaaa & aaaa & aaaa \\
\hline $\begin{array}{l}{ }^{*} A=A \text { or } A \\
\text { and } a=a \text { or } a\end{array}$ & & & & 1: 1 & & \\
\hline
\end{tabular}

Fig. 4. Genetic model describing the inheritance of resistance against Peronospora belbahrii in A, BCs2 F2 and B, BCs3 of a cross between the resistant tetraploid accession PI 500945 of Ocimum americanum var. americanum and the susceptible tetraploid $O$. basilicum 'Sweet basil'. 
dominant, one duplicate dominant, and one triplicate dominant genes) suggested an unusual model of 5:1 (R/S). The model indicates that resistance is controlled by a single duplicate dominant gene (Fig. 2). The model suggests that the resistant parent PI 500945 is tetraploid, carrying two copies of a dominant resistance gene $(A$ and $A^{\prime}$ ) on two homeologous chromosomes. The corresponding recessive alleles in the susceptible tetraploid parent Sweet basil are $a$ and $a^{\prime}$. The F1 $A A^{\prime}$ ' $a a^{\prime}$ produces six types of gametes: $A A^{\prime}, A a, A a^{\prime}$, $A^{\prime} a, A^{\prime} a^{\prime}$, and $a a^{\prime}$. The backcross of $\mathrm{F} 1$ to Sweet basil produces two phenotypes, $\mathrm{R}$ and $\mathrm{S}$, at a ratio of 5:1 (Fig. 2).

Because BCs1 plants were sterile, they were pollinated (backcrossed) with Sweet basil. In total, 22 second backcross generation to the susceptible parent (BCs2) progenies were obtained. BCs2 plants at the four- to six-leaf stage ( 8 to 46 plants/progeny) were

TABLE 5. Inheritance of resistance against downy mildew Peronospora belbahrii in 35 BCs 3 progenies derived from 11 BCs 2 progenies of the cross between resistant (R) accession Ocimum americanum var. americanum PI 500945 and susceptible (S) O. basilicum 'Sweet basil'

\begin{tabular}{|c|c|c|c|c|c|c|c|c|}
\hline \multirow[b]{2}{*}{ BCs1 } & \multirow[b]{2}{*}{ BCs $2 \times$ Sweet basil $(\mathrm{BCs} 3)$} & \multirow[b]{2}{*}{$N^{\mathrm{b}}$} & \multicolumn{2}{|c|}{ Observed ratio } & \multicolumn{2}{|c|}{ Expected ratio } & \multicolumn{2}{|c|}{$P^{\mathrm{a}}$} \\
\hline & & & $\mathrm{R}$ & $\mathrm{S}$ & $\mathrm{R}$ & $\mathrm{S}$ & for $1: 1$ & for $5: 1$ \\
\hline \multirow[t]{15}{*}{1} & 2 & 22 & 10 & 12 & 11 & 11 & 0.670 & $\ldots$ \\
\hline & 5 & 13 & 7 & 6 & 6.5 & 6.5 & 0.782 & $\ldots$ \\
\hline & 6 & 30 & 24 & 6 & 15 & 15 & $0.001 *$ & $0.624 * *$ \\
\hline & 12 & 8 & 3 & 5 & 4 & 4 & 0.480 & $\ldots$ \\
\hline & 14 & 39 & 21 & 18 & 19.5 & 19.5 & 0.631 & $\ldots$ \\
\hline & 15 & 15 & 11 & 4 & 7.5 & 7.5 & 0.071 & $\ldots$ \\
\hline & 16 & 29 & 15 & 14 & 14.5 & 14.5 & 0.853 & $\ldots$ \\
\hline & 19 & 18 & 4 & 14 & 9 & 9 & $0.019 * * *$ & $0.0000 * * *$ \\
\hline & 21 & 11 & 11 & 0 & 5.5 & 5.5 & $0.001^{*}$ & $0.117 * *$ \\
\hline & 22 & 37 & 21 & 16 & 18.5 & 18.5 & 0.411 & $\ldots$ \\
\hline & 23 & 11 & 7 & 4 & 5.5 & 5.5 & 0.366 & $\ldots$ \\
\hline & 24 & 30 & 13 & 17 & 15 & 15 & 0.465 & $\ldots$ \\
\hline & 27 & 13 & 10 & 3 & 6.5 & 6.5 & 0.052 & $0.53 * *$ \\
\hline & 29 & 62 & 28 & 34 & 31 & 31 & 0.446 & $\ldots$ \\
\hline & 30 & 33 & 13 & 20 & 16.5 & 16.5 & 0.22 & $\ldots$ \\
\hline \multirow[t]{4}{*}{2} & 1 & 25 & 13 & 12 & 12.5 & 12.5 & 0.841 & $\ldots$ \\
\hline & 4 & 18 & 8 & 10 & 9 & 9 & 0.637 & $\ldots$ \\
\hline & 8 & 30 & 14 & 16 & 15 & 15 & 0.715 & $\ldots$ \\
\hline & 11 & 20 & 10 & 10 & 10 & 10 & 1.000 & $\ldots$ \\
\hline \multirow[t]{3}{*}{3} & 1 & 22 & 16 & 6 & 11 & 11 & $0.033^{*}$ & $0.268 * *$ \\
\hline & 5 & 33 & 14 & 19 & 16.5 & 16.5 & 0.384 & $\ldots$ \\
\hline & 8 & 20 & 13 & 7 & 10 & 10 & 0.180 & $\ldots$ \\
\hline 4 & 1 & 4 & 1 & 3 & 2 & 2 & 0.317 & $\ldots$ \\
\hline \multirow[t]{4}{*}{6} & 5 & 9 & 2 & 7 & 4.5 & 4.5 & 0.096 & $\ldots$ \\
\hline & 11 & 6 & 4 & 2 & 3 & 3 & 0.414 & $\ldots$ \\
\hline & 12 & 22 & 12 & 10 & 11 & 11 & 0.670 & $\ldots$ \\
\hline & 18 & 15 & 8 & 7 & 7.5 & 7.5 & 0.796 & $\ldots$ \\
\hline 9 & 2 & 13 & 4 & 9 & 6.5 & 6.5 & 0.166 & $\ldots$ \\
\hline 10 & 4 & 6 & 3 & 3 & 3 & 3 & 1.000 & $\ldots$ \\
\hline 15 & 5 & 11 & 4 & 7 & 5.5 & 5.5 & 0.366 & $\ldots$ \\
\hline 17 & 3 & 25 & 10 & 15 & 12.5 & 12.5 & 0.317 & $\ldots$ \\
\hline 21 & 3 & 6 & 2 & 4 & 3 & 3 & 0.414 & $\ldots$ \\
\hline \multirow[t]{3}{*}{26} & 1 & 7 & 4 & 3 & 3.5 & 3.5 & 0.705 & $\ldots$ \\
\hline & 3 & 34 & 22 & 12 & 17 & 17 & 0.086 & $\ldots$ \\
\hline & 16 & 8 & 2 & 6 & 4 & 4 & 0.157 & $\ldots$ \\
\hline
\end{tabular}

a Asterisks: * indicates the model 1:1 unaccepted $(P<0.05), * *$ indicates the model 5:1 accepted $(P<0.05)$, and $* * *$ indicates both models unaccepted.

b Number of plants.

TABLE 6. Inheritance of resistance against downy mildew Peronospora belbahrii in large populations of BCs3 3 BCs 3 (self) and BCs4 families derived from the cross between parents resistant (R) accession Ocimum americanum var. americanum PI 500945 and susceptible (S) O. basilicum 'Sweet basil'

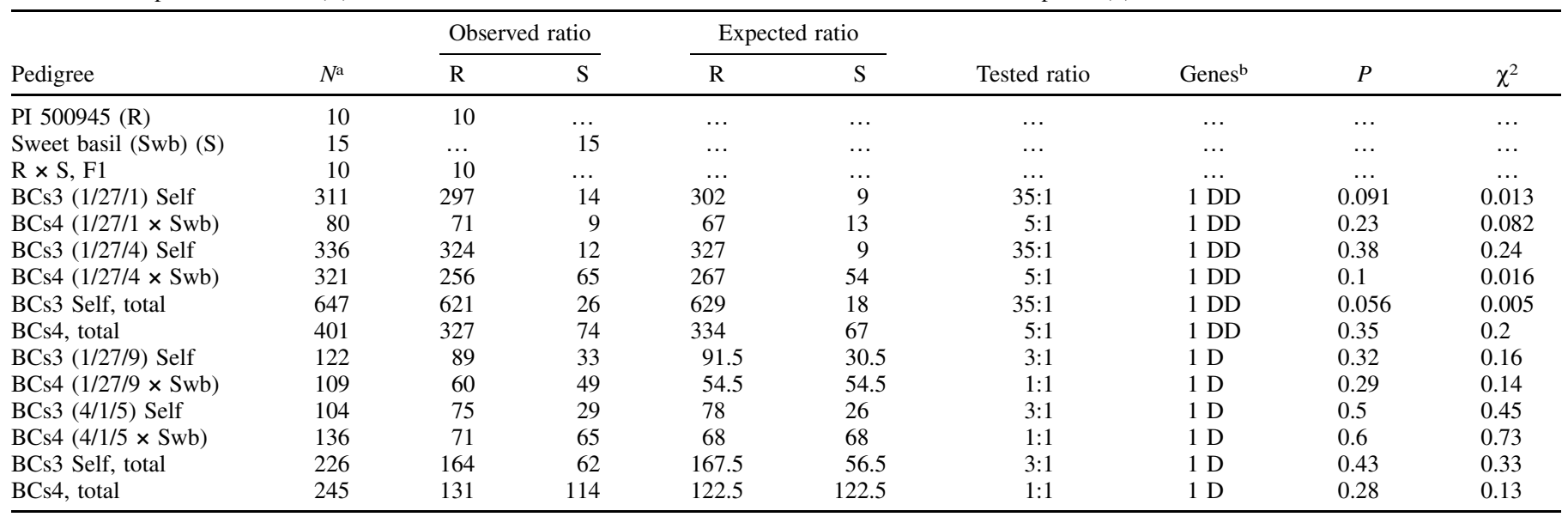

a Number of plants.

${ }^{\mathrm{b}} \mathrm{D}=$ dominant and $\mathrm{DD}=$ duplicate dominant. 
inoculated with $P$. belbahrii in growth chambers and their response to BDM was evaluated at $7 \mathrm{dpi}$. The results are presented in Table 3. Progenies showed one of two different modes of R/S segregation, either $5: 1$ or $1: 1$. The ratio between the two modes was $1: 4$ [(5:1):(1:1)].

A genetic model supporting the BCs 2 data presented in Table 3 is illustrated Figure 3. It shows that the backcross $A A^{\prime} a a^{\prime} \times a a^{\prime} a a^{\prime}$ yields a 5:1 R/S segregating progeny (Fig. 3A), whereas the other four backcrosses (Aaaa' $\times a a^{\prime} a a^{\prime}, A_{a} a a^{\prime} \times a a^{\prime} a a^{\prime}, A^{\prime} a a^{\prime} a^{\prime} \times a a^{\prime} a a^{\prime}$, and $A^{\prime} a a^{\prime} a^{\prime} \times a a^{\prime} a a^{\prime}$ ) yield 1:1 R/S segregating progenies (Fig. 3B). The backcross of the susceptible BCs1 $a a^{\prime} a^{\prime}$ a to Sweet basil $a a^{\prime} a^{\prime} a$ yields a susceptible progeny.

A single plant, BCs1-1, was fertile, enabling self-pollination $A A^{\prime} a a^{\prime} \times A A^{\prime} a a^{\prime}$. Its progeny plants segregated 35:1, R/S (Table 3, bottom), confirming that resistance is controlled by a duplicate dominant gene (Table 3; Fig. 3C).

All inoculated progeny plants were transplanted to a nethouse at $8 \mathrm{dpi}$ and disease records were taken at 1,2, and 3 months after transplanting. The response to disease as recorded in the growth chambers ( $\mathrm{R}$ or $\mathrm{S}$ ) was maintained in the field throughout the season.

BCs2 progeny plants segregated 19:16 fertile/sterile. The 19 fertile plants were both self-pollinated and backcrossed to Sweet basil. The 16 sterile plants were discarded.

In total, 18 of 19 self-pollinated BCs $2\left(A^{*} a * a a \times A^{*} a * a a\right)$ progenies segregated R/S at a ratio of 3:1 (Table 4; Fig. 4A), suggesting that a single dominant gene controls resistance in BCs2 plants. (Note that $A^{*}=A$ or $A^{\prime}$ and $a^{*}=a$ or a'.)

Of the 19 fertile plants, $16 \mathrm{BCs} 2$ plants were also backcrossed to Sweet basil $\left(A^{*} a^{*} a a \times a^{*} a^{*} a a\right)$ to obtain third backcross generation to the susceptible parent (BCs3) progenies. All BCs3 progenies segregated R/S at a ratio of 1:1 (Table 5; Fig. 4B), reaffirming that a single dominant gene controls resistance in BCs 2 plants. Four BCs 3 progenies segregated $\mathrm{R} / \mathrm{S}$ at a ratio of $5: 1$, probably because one homeologous chromosome (carrying resistance) has not yet replaced by a "susceptible" one.

Four single plants of four BCs3 families-1/27/1, 1/27/4, 1/27/9, and 4/1/5-were self-pollinated or backcrossed to Sweet basil and large offspring populations tested for response to BDM. BCs $3 \times \mathrm{BCs} 3$
$1 / 27 / 1$ and $1 / 27 / 4$ produced $621 \mathrm{R}$ and $26 \mathrm{~S}$ plants (35:1), whereas BCs $3 \times$ Sweet basil produced $327 \mathrm{R}$ and $74 \mathrm{~S}$ plants, confirming that each carries one duplicate dominant resistance gene (Table 6). $\mathrm{BCs} 3 \times \mathrm{BCs} 31 / 27 / 9$ and $4 / 1 / 5$ produced $164 \mathrm{R}$ and $62 \mathrm{~S}$ plants (3:1) whereas BCs $3 \times$ Sweet basil produced $131 \mathrm{R}$ and $114 \mathrm{~S}$ plants $(1: 1)$, confirming that each carries one dominant resistance gene (Table 6).

Compared with susceptibility of Sweet basil (Fig. 5, left), fourth backcross generation to the susceptible parent (BCs4) resistant lines that were subjected to an F2-F3 progeny test provided homozygous, BDMresistant lines (Fig. 5, right) exhibiting high yield and good aroma.

\section{DISCUSSION}

Downy mildew, caused by $P$. belbahrii, is currently the most destructive disease of sweet basil worldwide. No resistant cultivars are available. The aim of the present study was to incorporate genetic resistance into sweet basil, to supply farmers with relief from the disease.

In a previous study (Ben-Naim et al. 2015b), we reported that sweet basil $(O$. basilicum) cultivars carry no resistance against downy mildew but wild basil species exhibit profound resistance. To meet our aim, we choose $O$. americanum var. americanum PI 500945 as a source of resistance due to the dominant nature of its resistance. Microscopic examinations revealed that resistance resulted from an early cessation of the pathogen's development in the penetrated epidermal cell that was associated with a hypersensitive response. Susceptible Sweet basil was selected as a recurrent parent due to its elite aroma and excellent yield properties.

F1 interspecific hybrid plants derived from the cross PI $500945 \times$ Sweet basil were fully resistant to downy mildew, as was PI 500945, but sterile, a serious obstacle to further exploiting this resistance. Sterility was both male and female. Our flow cytometry data, as well as those of Koroch et al. (2010) and Rewers and Jedrzejczyk (2016), revealed that both parents are tetraploid. The fact that the F1 was sterile indicated a lack of chromosomal homology between the parents (homeology).

\section{Susceptible, "Sweet basil" Resistant, BCs4F3}

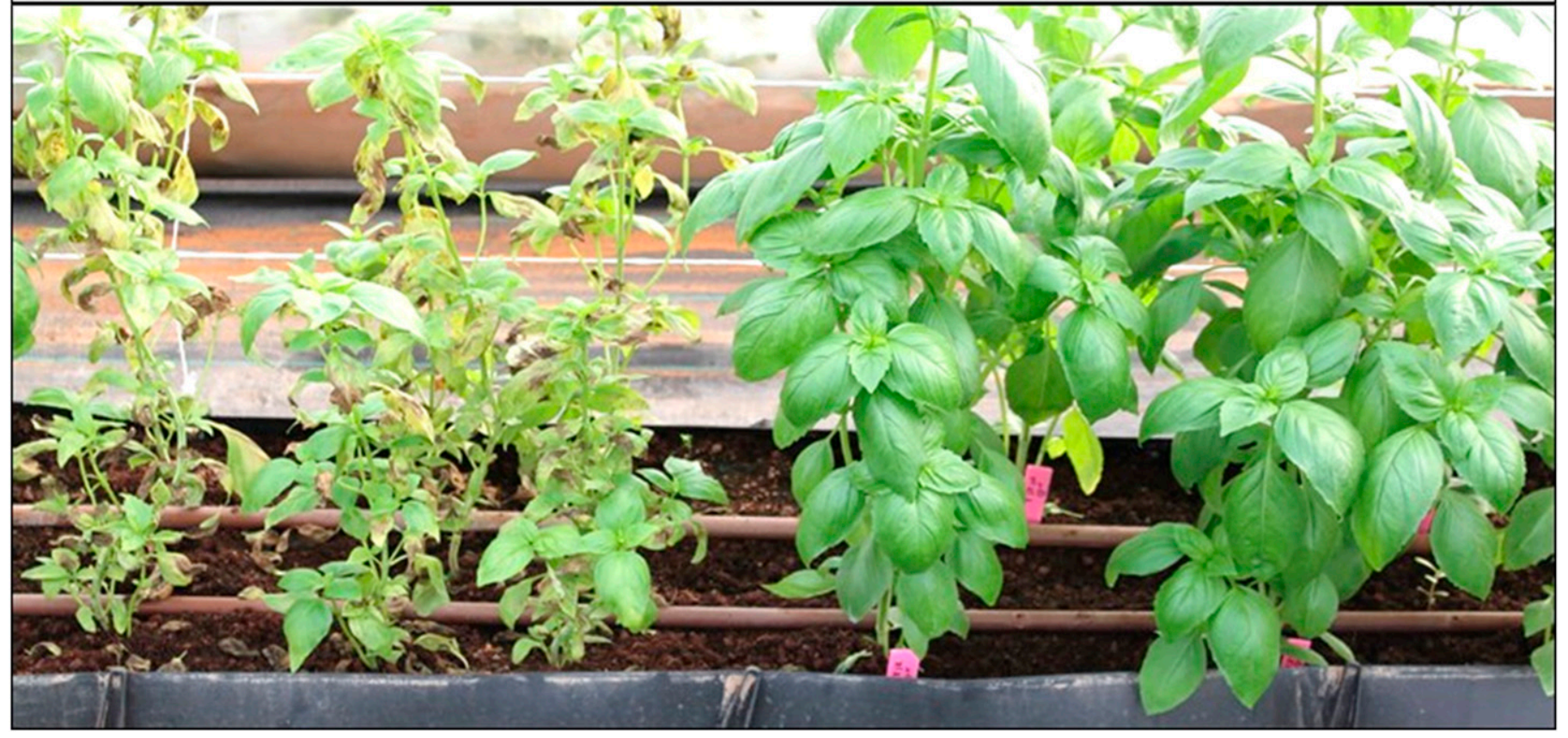

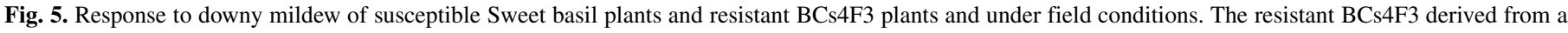
cross between the resistant tetraploid accession PI 500945 of Ocimum americanum var. americanum and the susceptible tetraploid O. basilicum 'Sweet basil'. 
We overcame the sterility barrier of F1 Ocimum interspecific hybrid plants by exploiting the technology of embryo rescue (Doganlar et al. 1997). Interspecific $\mathrm{F} 1$ hybrids may produce abnormal, nondisjuncted gametes. Such gametes suffer chromosomal imbalance but may still be fertilized with normal gametes (Morales and Dujon 2012). In vitro embryo rescue techniques may enable the development of fertilized abnormal gametes into plantlets. Embryo rescue in Datura stramonium allowed development of plantlets from nondisjunction gametes after fertilization (Amiri and Kazemitabar 2011).

In-spite of the very low rate of success $(0.4 \%)$, we were able to produce 115 BCs1 plants ([PI $500945 \times$ Sweet basil] $\times$ Sweet basil), of which 100 were fully resistant and 15 were susceptible to BDM. This unusual 5:1 segregation ratio was analyzed for fit to one dominant, two dominant, one duplicate dominant, and one triplicate dominant genes model, corresponding to $2 n, 2 n, 4 n$, or $6 n$ ploidy levels, respectively. The one duplicate dominant gene model $(2 n=4 \mathrm{x})$ was accepted, confirming that parents are tetraploid and suggesting that a duplicate dominant gene (designated $\mathrm{PblA}$ and $\mathrm{PblA}{ }^{\prime}[\mathrm{Pb}$ for $P$. belbahrii]) controls resistance against BDM in BCs1 plants. $P b 1$ is probably located on two homeologous chromosomes, as expected for a tetraploid resistant parent. The 5:1 ratio obtained in the BCs1 population indicates that, in spite of the genomic differences that exist between the two species (sterility of F1), the two subgenomes of $O$. basilicum and the two subgenomes of $O$. americanum var. americanum undergo pairing to produce combined gametes.

Our inheritance model (Fig. 2) corroborates that of $\mathrm{Wu}$ et al. (2001), who predicted that, in a cross between tetraploid species, six new pair combinations of homeologous chromosomes occur in the gametes of F1. Scheid et al. (2003) showed that, for a duplicate dominant gene, such six-pair combinations produce, upon selfpollination, a pedigree that segregates at a ratio of 35:1.

Newly force-formed interspecific hybrids suffer rapid changes in the genetic contribution of each parent, including chromosome loss or aneuploidy, translocations, and loss of heterozygosity (Griffiths et al. 2002). Usually, nondisjunction gametes lead to aneuploidy, including nullisomy $(2 n-2)$, monosomy $(2 n-1)$, trisomy $(2 n+1)$, or tetrasomy $(2 n+2)$. In most cases, aneuploidy leads to sterility, less so in trisomy. Chromosome pairing in meiotic cells of trisomic plants may form trivalent paring, which may develop stable and fertile progenies by eliminating the added chromosome. In tetrasomy, chromosomes may form regular bivalent paring, leading to infertility, or multivalent chromosome paring, which may lead to creation of a new species with an added pair of new chromosomes. Nullisomy and monosomy are less common because of loss of functionality (Griffiths et al. 2002).

The cross between F1 $\times$ Sweet basil segregated 100:15 R/S in BCs1 plants. All 31 resistant plants we choose for producing BCs2 were either fully or partially fertile. Their fertility rejects the possibility that their resistance derived from aneuploidy.

Flow cytometry studies showed that $O$. basilicum as well as O. americanum PI 500945 are tetraploid (Koroch et al. 2010; Rewers and Jedrzejczyk 2016). Allotetraploid species, carrying two different homeologous sets of chromosomes, occur in nature (Adams and Wendel 2005). We named the resistance genes in O. americanum PI $500945 \mathrm{PblA}$ and $\mathrm{PblA}$ ' and the null alleles in Sweet basil $p b l a$ and pbla'. We show here that the duplicate resistance gene $P b 1 A$ and $P b 1 A^{\prime}$ from $O$. americanum was incorporated into the genome of $O$. basilicum. This could have happened by replacement of one or two chromosomes on which the $\mathrm{Pbl}$ gene resides with the homeologous chromosome of $O$. basilicum, or by crossing-over introgression of a segment on which the $\mathrm{Pbl}$ locus resides into the homeologous chromosome of $O$. basilicum.

BCs 2 offspring plants ([\{PI $500945 \times$ Sweet basil $\} \times$ Sweet basil $] \times$, Sweet basil') showed two modes of R/S segregation, 5:1 and 1:1, at a ratio of 4:1 (Fig. 3). Such modes of segregation corroborate a model of two homeologous chromosomes, both derived from the tetraploid resistant parent, and each carries a single dominant resistance gene. The ratio of $4: 1$ between the two modes of segregation makes it unlikely that two genes residing on one chromosome are responsible for resistance.
BCs2 plants showed restored fertility and, therefore, were selfpollinated to obtain BCs2-F2 progenies. All F2 progenies obtained, except one, segregated $3: 1 \mathrm{R} / \mathrm{S}$, suggesting that a single dominant gene controls resistance in $\mathrm{BCs} 2$. The restoration of fertility and the change in the mode of inheritance indicated that one of the two homeologous chromosomes derived from the tetraploid resistant parent has been replaced with a chromosome from the susceptible parent after the third backcross to Sweet basil.

The introgression of the resistance gene from $O$. americanum into $O$. basilicum indicates that the homeologous chromosomes of the two species are related. This enabled the introgression of the resistance gene and restoration of fertility.

Currently, there is only one report on the inheritance of resistance against BDM in basil. The commercial basil cultivar Mrihani (MR1) was used as a resistance source at Rutgers University (Pyne et al. 2015). MRI (resistant parent $=$ P1) was crossed with cultivar SB22 (susceptible parent $=\mathrm{P} 2$ ) and evaluated for resistance against BDM in $\mathrm{F} 1, \mathrm{~F} 2, \mathrm{BC} 1 \mathrm{P} 1$, and BC1P2. Two genes with digenic epistasis were found to control resistance in this family. The reported resistance was high but not complete. These efforts of combining resistance genes against BDM into cultivated basil will improve yield and product quality and enable organic growth without the use or with less use of fungicides.

Our current efforts are devoted to discovering the nature of $\mathrm{PblA} /$ $P b 1 A^{\prime}$ from PI 500945, mapping them into the genome of basil, and searching for their product proteins.

\section{ACKNOWLEDGMENTS}

This research was supported by Research Grant Number US-4947-16 R from the United States-Israel Binational Agricultural Research and Development Fund.

\section{LITERATURE CITED}

Adams, K. L., and Wendel, J. F. 2005. Polyploidy and genome evolution in plants. Curr. Opin. Plant Biol. 8:135-41.

Aghaei, M., Darvishzadeh, R., and Hassani, A. 2012. Molecular characterization and similarity relationships among Iranian basil (Ocimum basilicum L.) accessions using inter simple sequence repeat markers. Rev. Cienc. Agron. 43:312-320.

Amiri, S., and Kazemitabar, S. K. 2011. Enhancement of callus induction and regeneration efficiency from embryo cultures of Datura stramonium by adjusting carbon sources and concentrations. African J. Biotechnol. 10: 10101-10107.

Arumuganathan, K., and Earle, E. D. 1991. Estimation of nuclear DNA content of plants by flow cytometry. Plant Mol. Biol. Report. 9:229-241.

Babadoost, M. 2010. Downy mildew of basil in Illinois. (Abstr.) Phytopathology 100:S9.

Ben-Naim, Y., Falach, L., and Cohen, Y. 2015a. Resistance to Peronospora belbahrii in wild Ocimum species and its introgression into 'Sweet basil'. Phytoparasitica 43:371.

Ben-Naim, Y., Falach, L., and Cohen, Y. 2015b. Resistance against basil downy mildew in Ocimum species. Phytopathology 105:778-785.

Ben-Naim, Y., Falach, L., and Cohen, Y. 2016. Transfer of resistance against downy mildew from wild basil to 'Sweet basil'. Phytoparasitica 44:275.

Carović-Stanko, K., Liber, Z., Besendorfer, V., Javornik, B., Bohanec, B., Kolak, I., and Satovic, Z. 2010. Genetic relations among basil taxa (Ocimum L.) based on molecular markers, nuclear DNA content, and chromosome number. Plant Syst. Evol. 285:13-22.

Che, P., Lall, S., Nettleton, D., and Howell, S. H. 2006. Gene expression programs during shoot, root, and callus development in Arabidopsis tissue culture. Plant Physiol. 141:620-637.

Cisneros, A., and Tel-Zur, N. 2010. Embryo rescue and plant regeneration following interspecific crosses in the genus Hylocereus (Cactaceae). Euphytica 174:73-82.

Cohen, Y., and Ben-Naim, Y. 2016. Nocturnal fanning suppresses downy mildew epidemics in 'Sweet basil'. PLoS One 11:e0155330.

Cohen, Y., and Rubin, A. E. 2015. Daytime solar heating controls downy mildew Peronospora belbahrii in 'Sweet basil'. PLoS One 10:e0126103.

Cohen, Y., Vaknin, M., Ben-Naim, Y., and Rubin, A. E. 2013a. Light suppresses sporulation and epidemics of Peronospora belbahrii. PLoS One 8:e81282. 
Cohen, Y., Vaknin, M., Ben-Naim, Y., Rubin, A. E., Galperin, M., Silverman, D., Bitton, S., and Adler, U. 2013b. First report of the occurrence and resistance to mefenoxam of Peronospora belbahrii, causal agent of downy mildew of basil (Ocimum basilicum) in Israel. Plant Dis. 97:692.

Doganlar, S., Frary, A., and Tanksley, S. D. 1997. Production of interspecific F-1 hybrids, BC1, BC2 and BC3 populations between Lycopersicon esculentum and two accessions of Lycopersicon peruvianum carrying new rootknot nematode resistance genes. Euphytica 95:203-207.

Dolezel, J., Bartos, J., Voglmayr, H., and Greilhuber, J. 2003. Nuclear DNA content and genome size of trout and human. Cytometry 51A:127-128.

Doležel, J., Greilhuber, J., and Suda, J. 2007. Estimation of nuclear DNA content in plants using flow cytometry. Nat. Protoc. 2:2233-2244.

Eeckhaut, T., Van Laere, K., De Riek, J., and Van Huylenbroeck, J. 2006. Overcoming interspecific barriers in ornamental plant breeding. In: Floriculture, Ornamental and Plant Biotechnology: Advances and Topical Issues. Global Science Books, London.

Farahani-Kofoet, R. D., Römer, P., and Grosch, R. 2012. Systemic spread of downy mildew in basil plants and detection of the pathogen in seed and plant samples. Mycol. Prog. 11:961-966.

Farahani-Kofoet, R. D., Römer, P., and Grosch, R. 2014. Selecting basil genotypes with resistance against downy mildew. Sci. Hortic. (Amsterdam) 179:248-255.

Gamborg, O. L., Miller, R. A., and Ojima, K. 1968. Nutrient requirements of suspension cultures of soybean root cells. Exp. Cell Res. 50:151-158.

Gilardi, G., Demarchi, S., Garibaldi, A., and Gullino, M. L. 2013. Management of downy mildew of 'Sweet basil' (Ocimum basilicum) caused by Peronospora belbahrii by means of resistance inducers, fungicides, biocontrol agents and natural products. Phytoparasitica 41:59-72.

Griffiths, A. J. F., Gelbart, W. M., Lewontin, R. C., and Miller, J. H. (eds.) 2002. Modern Genetic Analysis: Integrating Genes and Genomes, 2nd ed. W. H. Freeman, New York.

Harley, R. M., and Heywood, C. A. 1992. Chromosome numbers in tropical American Labiatae. In: Advances in Labiatae Science. R. M. Harley and T. Reynolds, eds. Royal Botanic Gardens, London.

Homa, K., Barney, W. P., Ward, D. L., Wyenandt, C. A., and Simon, J. E. 2014. Evaluation of fungicides for the control of Peronospora belbahrii on 'Sweet basil' in New Jersey. Plant Dis. 98:1561-1566.

Ikeda-Iwai, M., Umehara, M., Satoh, S., and Kamada, H. 2003. Stress-induced somatic embryogenesis in vegetative tissues of Arabidopsis thaliana. Plant J. 34:107-114

Koroch, A. R., Wang, W., Michael, T. P., Dudai, N., Simon, J. E., and Belanger, F. C. 2010. Estimation of nuclear DNA content of cultivated Ocimum species by using flow cytometry. Isr. J. Plant Sci. 58:183-189.

McGrath, M. T. 2016. Efficacy of fungicides, biopesticides, and resistant varieties for managing downy mildew in basil. (Abstr.) Phytopathology 106:S2.4.

Mehetre, S. S., and Aher, A. R. 2004. Embryo rescue: A tool to overcome incompatible interspecific hybridization in Gossypium Linn. A review. Int. J. Biotechnol. 3:29-36.

Mersha, Z., Zhang, S., Fu, Y., Mo, X., Raid, R. N., and Hau, B. 2013. Efficacy of acibenzolar-S-methyl and beta-aminobutyric acid for control of downy mildew in greenhouse grown basil and peroxidase activity in response to treatment with these compounds. J. Phytopathol. 161:154-164.

Mersha, Z., Zhang, S. A., and Raid, R. N. 2012. Evaluation of systemic acquired resistance inducers for control of downy mildew on basil. Crop Prot. 40:83-90.

Miyajima, D. 2006. Ovules that failed to form seeds in zinnia (Zinnia violacec Cav). Sci. Hortic. (Amsterdam, Neth.) 107:176-182.

Morales, L., and Dujon, B. 2012. Evolutionary role of interspecific hybridization and genetic exchanges in yeasts. Microbiol. Mol. Biol. Rev. 76: 721-739.

Morton, J. K. 1962. Cytotaxonomic studies on the west African Labiatae. J. Linn. Soc. London, Bot. 58:231-283.

Mukherjee, M., Datta, A. K., and Maiti, G. G. 2005. Chromosome number variation in Ocimum basilicum L. Cytologia (Tokyo) 70:455-458.

Murashige, T., and Skoog, F. 1962. A revised medium for rapid growth and bio assays with tobacco tissue cultures. Physiol. Plant. 15:473-497.
Patel, J. S., Zhang, S., and de Novaes, M. I. C. 2014a. Oxathiapiprolin: An effective new chemistry for control of downy mildew of basil. (Abstr.) Phytopathology 104:S3.90.

Patel, J. S., Zhang, S., and de Novaes, M. I. C. 2014b. Effect of plant age and Acibenzolar-S-methyl on development of downy mildew of basil. HortScience 49:1392-1396

Paton, A., and Putievsky, E. 1996. Taxonomic problems and cytotaxonomic relationships between and within varieties of Ocimum basilicum and related species (Labiatae). Kew Bull. 51:509-524.

Paton, A. L. A. N., Harley, R. M., and Harley, M. M. 1999. Ocimum: An overview of classification and relationships. Pages 1-38 in: Basil: the Genus Ocimum. R. Hiltunen and Y. Holm, eds. Harwood Academic, Amsterdam.

Pintore, I., Gilardi, G., Gullino, M. L., and Garibaldi, A. 2016. Detection of mefenoxam-resistant strains of Peronospora belbahrii, the causal agent of basil downy mildew, transmitted through infected seeds. Phytoparasitica 44:563-569.

Pushpangadan, P., and Sobti, S. N. 1982. Cytogenetical studies in the genus Ocimum. I. Origin of $O$. americanum, cytotaxonomical and experimental proof. Cytologia (Tokyo) 47:575-583.

Pushpangadan, P., Sobti, S. N., and Khan, R. 1975. Karyomorphological studies in the genus Ocimum: 1. Basilicum group. Nucleus 18:177-182.

Pyne, R. M., Koroch, A. R., Wyenandt, C. A., and Simon, J. E. 2015. Inheritance of resistance to downy mildew in Sweet basil. J. Am. Soc. Hortic. Sci. 140: 396-403.

Raid, R. N., Roberts, P. D., Harmon, P. F., Palmateer, A. J., and Jordan, S. A. 2010. Basil downy mildew in Florida: A disease of new importance. (Abstr.) Phytopathology 100:S175.

Rewers, M., and Jedrzejczyk, I. 2016. Genetic characterization of Ocimum genus using flow cytometry and inter-simple sequence repeat markers. Ind. Crops Prod. 91:142-151.

Rodrangboon, P., Pongtongkam, P., Suputtitada, S., and Adachi, T. 2002. Abnormal embryo development and efficient embryo rescue in interspecific hybrids, Oryza sativa $\times$ O. minuta and $O$. sativa $\times O$. officinalis. Breed. Sci. 52:123-129.

Sage, T. L., Strumas, F., Cole, W. W., and Barrett, S. C. H. 1999. Differential ovule development following self- and cross-pollination: The basis of self-sterility in Narcissus triandrus (Amaryllidaceae). Am. J. Bot. 86: $855-870$.

Sairkar, P., Vijay, N., Silawat, N., Garg, R. K., Chouhan, S., Batav, N., and Mehrotra, N. N. 2012. Inter-species association of Ocimum genus as revealed through random amplified polymorphic DNA fingerprinting. Sci. Secure J. Biotechnol. 1:1-8.

Scheid, O. M., Afsar, K., and Paszkowski, J. 2003. Formation of stable epialleles and their paramutation-like interaction in tetraploid Arabidopsis thaliana. Nat. Genet. 34:450-454.

Simon, J. E., Quinn, J., and Murray, R. G. 1990. Basil-A source of essential oils. Pages 484-489 in: Advances in New Crops Research. J. Janick and J. E. Simon, eds. Timber Press, Portland, OR.

Trigiano, R. N., and Gray, D. J. 2011. Plant Tissue Culture, Development, and Biotechnology. CRC Press, Boca Raton, FL.

Vaarama, A. 1947. Some chromosome numbers in the genera Angelica, Ocimum, Satureja, Thymus and Cnicus. Arch. Soc. Zool. Bot. Fenn. "Vanamo" 2:55-59.

Wu, R., Gallo-Meagher, M., Littell, R. C., and Zeng, Z. B. 2001. A general polyploid model for analyzing gene segregation in outcrossing tetraploid species. Genetics 159:869-882.

Wyenandt, C. A., Simon, J. E., McGrath, M. T., and Ward, D. L. 2010. Susceptibility of Basil cultivars and breeding lines to downy mildew (Peronospora belbahrii). HortScience 45:1416-1419.

Wyenandt, C. A., Simon, J. E., Pyne, R. M., Homa, K., McGrath, M. T., Zhang, S. A., Raid, R. N., Ma, L. J., Wick, R., Guo, L., and Madeiras, A. 2015. Basil downy mildew (Peronospora belbahrii): Discoveries and challenges relative to its control. Phytopathology 105:885-894.

Zhang, X. P., Rhodes, B. N., and Whitesides, J. F. 1994. Determination of watermelon ploidy level using flow cytometry. Cucurbit Genet. Coop. Rep. 17:102-105 